\title{
Why We Should Care About Ebola in West Africa and Middle East Respiratory Syndrome in South Korea: Global Health Ethics and the Moral Insignificance of Proximity
}

\author{
Benedict Shing Bun Chan • Zion Tsz Ho Tse • \\ King-Wa Fu • Chi-Ngai Cheung • \\ Isaac Chun-Hai Fung
}

Received: 16 August 2015 / Accepted: 26 November 2015 / Published online: 10 December 2015

(C) Journal of Bioethical Inquiry Pty Ltd. 2015

In the era of globalization, no society exists in isolation. Global transportation networks facilitate the international spread of emerging infectious diseases, such as Ebola and Middle East Respiratory Syndrome (MERS). From restrictions of travel with regard to Ebola-stricken countries to international aid delivered to West Africa, from advice against travelling to South Korea (The Government of Hong Kong Special Administrative Region 2015) to experts from the World Health Organization visiting Seoul, decisions made by any country

B. S. B. Chan $(\bowtie)$

Department of Religion and Philosophy, Hong Kong Baptist University, Kowloon Tong, Hong Kong

e-mail: benedictchan@hkbu.edu.hk

Z. T. H. Tse

College of Engineering, The University of Georgia, Athens,

Georgia, USA

e-mail: ziontse@uga.edu

\section{K.-W. Fu}

Journalism and Media Studies Centre, The University of Hong Kong, Pok Fu Lam, Hong Kong

e-mail:kwfu@hku.hk

\section{C.-N. Cheung}

Department of Psychology, Emory University, Atlanta, Georgia, USA

e-mail: chi-ngai.cheung@emory.edu

\section{C.-H. Fung}

Department of Epidemiology, Jiann-Ping Hsu College of Public Health, Georgia Southern University, Statesboro, Georgia, USA e-mail: cfung@georgiasouthern.edu often have global health ramifications. Global health advocates affirm the importance of moral responsibilities for global public health. However, does everyone have moral responsibilities to help stop the Ebola outbreak in West Africa or MERS in South Korea and the Middle East? Should we consider global health issues to be as important as domestic ones?

Let us take the 2014 Ebola outbreak as an example. Medical and public health professionals agree that we should help stop the Ebola outbreak in West Africa, both to aid people in those countries and to prevent future imported cases into other countries, including the United States. However, despite the dire consequences of the Ebola outbreak, many Americans seemed unconcerned about it until the first domestic case was diagnosed in the United States. Social media and search engine data provide us with some evidence for this observation. In October 2014, the number of tweets about Ebola in ther United States was 1,561 times the number of those about Ebola in West Africa (The Lancet 2014). Global Twitter and Google traffic about Ebola increased sharply after the first U.S. domestic Ebola case, and many Ebola-related tweets were associated with negative emotions such as anxiety (Fung et al. 2014). Research shows that negative emotions are "contagious" via social media (Kramer, Guillory, and Hancock 2014). Nevertheless, such emotional reactions only appeared when Ebola hit home. The volume of Ebola-related tweets dropped rapidly once the domestic cases were resolved (Fung et al. 2014).

Similarly, our prior study comparing Chinese social media users' reactions to the MERS outbreak in the 
Middle East in 2012 and the influenza A (H7N9) outbreak in China in 2013 suggests that people are more concerned about outbreaks at home than those abroad (Fung et al. 2013). This difference is important to global health advocates because moral responsibilities and resource allocation are intertwined in public debates. Global health advocates' arguments may fail to convince those who do not share the moral conviction that everyone has a responsibility for global health issues. A proposal to allocate resources to stop an outbreak as far away as West Africa will only work if we can establish a consensus that we have a moral responsibility to help end this health crisis.

To do so, we need to develop and promote global health ethics. Global health ethics is a discipline concerning the normative and moral questions of global health (Stapleton et al. 2014). Although global health ethicists may dispute over which moral theory is the best, they all share a common view that we have moral responsibilities for people throughout the world, just like we have moral responsibilities for people in our own society. For example, Peter Singer, a Utilitarian, argues that we have a moral responsibility to stop bad things from happening if doing so does not cost us something comparably morally significant. Singer further argues that based on such a principle, proximity is irrelevant to our moral considerations (Singer 1972). Ethicists of care argue that caring relations are important in ethics, and they realize that care work is a fundamental component of public health. Although they disagree with Singer's Utilitarianism, they agree that the Ethics of Care must be global, instead of private. They also argue that the globalization of caring relations would help to create a peaceful and caring environment for everyone in the world (Robinson 2011).

Global health ethics can be applied to practical issues in public health. As the Ebola epidemic unfolded in Guinea, Liberia, and Sierra Leone, human rights issues appeared along with public health challenges. There has been tension and mistrust between the affected local populations and healthcare workers, as trust in government authorities is low. Mass quarantine of forest areas, urban slums, or even the whole group of countries would have violated human rights such as freedom of movement and liberty and security, while being ineffective to curtail the epidemic (Eba 2014). However, as in the case of the South Korean who defied the imposed voluntary home quarantine and travelled to mainland China via Hong Kong and tested positive for MERS in a hospital in Huizhou (Hui, Perlman, and Zumla 2015). sometimes the curtail of an individual's rights in order to protect the health of the community may be justified. Global health ethics teaches us what moral considerations we should have in these cases. Likewise, many people rely on cultural and religious narratives to make sense of what is life-threatening and seemingly uncontrollable (Kiser and Santibañez 2014). It has been argued from the philosophical perspective that concepts of human rights are universal and compatible with different cultures and religions (Chan 2014). Therefore, we should treat the Ebola patients in West Africa and MERS patients in South Korea the same as patients anywhere else. Global health ethics also teaches us how to reply to the challenge that we cannot afford to help everyone in the world. For example, Singer famously argues that we should help patients in West Africa and South Korea unless the cost is something comparably morally significant. Some also argue that the Ethics of Care provides a moral foundation to global security and public health, and so we should consider human security issues through the lens of the Ethics of Care (Robinson, 2011).

While there is still room for improvement in current global health ethics theories, these theories can help us establish a moral consensus for improving global health. With better education in global health ethics, we can bridge the gap between the ideal world and reality. A well-informed general public will be our best supporters in advocacy for more public and private funding for curtailing the Ebola outbreak in West Africa or MERS outbreak in South Korea and for global health at large.

Acknowledgements We thank Dr. Michelle Tsz-Wan Ho, Ms. Winny S.C. Kwan, and Dr. Scott Santibañez for their comments on previous versions of this manuscript.

\section{Compliance with ethical guidelines}

Conflicts of Interest We declare that we have no conflicts of interest.

Disclaimer/Funding Statement I. C.-H. Fung receives salary support from the Centers for Disease Control and Prevention (CDC) (15IPA1509134). This letter is not related to Fung's CDC-supported research. The CDC had no role in the writing and submission of this letter. This letter does not represent the official positions of the CDC or the United States Government. 


\section{References}

Chan, B.S.B. 2014. A human rights debate on physical security, political liberty, and the Confucian tradition. Dao: A Journal of Comparative Philosophy 13(4): 567-588.

Eba, P.M. 2014. Ebola and human rights in West Africa. The Lancet 384(9960): 2091-2093.

Fung, I.C.-H., K.-W. Fu, Y. Ying, B. Schaible, Y. Hao, C.-H. Chan, and Z.T.-H. Tse. 2013. Chinese social media reaction to the MERS-CoV and avian influenza A (H7N9) outbreaks. Infectious Diseases of Poverty 2(1): 31. doi:10.1186/20499957-2-31.

Fung, I.C.-H., Z.T.H. Tse, C.-N. Cheung, A.S. Miu, and K.-W. Fu. 2014. Ebola and the social media. The Lancet 384(9961): 2207.

Hui, D.S., S. Perlman, and A. Zumla. 2015. Spread of MERS to South Korea and China. The Lancet: Respiratory medicine 3(7): 509-510.

Kiser, M., and S. Santibañez. 2014. Influenza pandemic. In Religion as a Social determinant of public health, edited by E.L. Idler, 382-395. New York: Oxford University Press.

Kramer, A.D.I., J.E. Guillory, and J.T. Hancock. 2014. Experimental evidence of massive-scale emotional contagion through social networks. Proceedings of the National Academy of Sciences of the United States of America 111(24): 8788-8790.

Robinson, F. 2011. The ethics of care: A feminist approach to human security. Philadelphia: Temple University Press.

Singer, P. 1972. Famine, affluence, and morality. Philosophy \& Public Affairs 1(1): 229-243.

Stapleton, G., P. Schröder-Bäck, U. Laaser, A. Meershoek, and D. Popa. 2014. Global health ethics: An introduction to prominent theories and relevant topics. Global Health Action 7: 23569. doi:10.3402/gha.v7.23569.

The Government of Hong Kong Special Administrative Region. 2015. SFH, DoH and Controller of CHP on MERS. http:// www.info.gov.hk/gia/general/201506/08/P201506080793. htm. Accessed July 28, 2015.

The Lancet. 2014. The medium and the message of Ebola. The Lancet 384(9955): 1641. 